\title{
Dynamic rupture propagation on a 2D fault with fractal frictional properties
}

\author{
Satoshi Ide \\ Department of Earth and Planetary Science, University of Tokyo, Japan \\ (Received March 27, 2007; Revised August 30, 2007; Accepted September 12, 2007; Online published October 19, 2007)
}

\begin{abstract}
We study dynamic rupture propagation on a 2D fault with a spatially heterogeneous slip-weakening friction law under a homogeneous stress condition. The slip-weakening distance $D_{c}$ is determined based on fault topography, characterized by the fractal dimension $\delta$ and a normalized slip-weakening distance $D_{c 0}$. Assuming different combination of these parameters, we carry out a large number of dynamic rupture simulations to discuss event size statistics and the scaling relations of macroscopic parameters such as seismic moment, rupture velocity, seismic energy, and radiation efficiency. All stopped events obey an approximately power-law size-number relation. However, statistically self-similar rupture propagation is observed only for selfsimilar fault topography with $\delta=1$, where the average rupture velocity is controlled by $D_{c 0}$. This suggests that a power-law sizenumber relation does not simply mean the self-similarity of rupture process. Both upper and lower fractal limits in fault topography disturb the power-law statistics and the lower fractal limit results in excess of events of the characteristic size. The facts that slow ruptures can radiate large seismic energy and that fast ruptures are not always efficient suggest the importance of local acceleration, deceleration, and arrest of rupture. We also show that the spatial regularity of slip-weakening distance is essential to make the above results.
\end{abstract}

Key words: Dynamic rupture, fractal topography, self-similarity, seismic energy, rupture propagation velocity.

\section{Introduction}

One important aspect of earthquake is similarity of phenomena in wide scale range. Since Kanamori and Anderson (1975) pointed out geometrical similarity of earthquake source, many studies have confirmed that most earthquakes are similar process with scale invariants such as stress drop and scaled energy, the ratio between seismic energy and seismic moment. Although it is still controversial whether the scaled energy is constant in wide scale range (e.g. Ide and Beroza, 2001; Walter et al., 2006; Yamada et al., 2007), the change is small, within 1-2 order, for the difference of seismic moment by 5-10 orders.

Earthquakes occur as dynamic ruptures, frictional slip with fracture, on fault planes, which contain various lengths of heterogeneity. Okubo and Aki (1987) found fractal structure of fault traces, while Brown and Scholz (1985) and Power et al. (1987) found that the topography of fracture surfaces has power law spectra with exponents from 2 to 3 . Recently, more thorough studies on fault topography have been carried out by wide-scale mapping of exposed fault surfaces. Renard et al. (2006) found the difference of fractal topography depending on the angle to the slip vector. Sagy et al. (2007) also found difference of topography depending on the angle to the slip vector in the broad range from $10 \mu \mathrm{m}$ to $120 \mathrm{~m}$, and concluded that repeating fault slip generates characteristic size in the power spectrum in the slip direction, which evolves as the cumulative slip increases. While fault slip produces characteristic structures

Copyright (c) The Society of Geomagnetism and Earth, Planetary and Space Sciences (SGEPSS); The Seismological Society of Japan; The Volcanological Society of Japan; The Geodetic Society of Japan; The Japanese Society for Planetary Sciences; TERRAPUB. in the fault topography, the fault topography also controls the behavior of dynamic rupture propagation.

There have been many studies that dealt with dynamic rupture process on a fault plane with heterogeneous properties. The first one was dynamic rupture simulations on a planer fault with separated high prestress areas by Day (1982). More realistic rupture processes have been modeled based on the heterogeneous distribution of stress and the assumption of a friction law, which is usually the slip-weakening law (e.g., Olsen et al., 1997; Aochi and Fukuyama, 2002; Guatteri et al., 2003). Ripperger et al. (2006) provided a comprehensive study assuming various fractal stress distributions with a uniform slip-weakening distance. They demonstrated that the heterogeneity of stress yields earthquakes of a limited size range. Although the complex distribution of stress does controls earthquake rupture process, the values of stress are not likely to change greatly between small and large earthquakes, and the difference of stress may be insufficient to explain the similarity and difference between large and small earthquakes. One difficulty of a heterogeneous stress model with a homogeneous slip-weakening distance is that there exists an inherent size above which rupture cannot be arrested.

It is well known from the linear fracture mechanics that the onset and propagation of rupture are controlled by fracture energy. For example, the size of the critical crack in a homogeneously stressed medium is proportional to the fracture energy. The propagation velocity of rupture front is determined by energy balance between crack extension force and fracture energy (e.g. Freund, 1998). The value of fracture energy is on the order of $1 \mathrm{~J} / \mathrm{m}^{2}$ in the rock fracture/friction experiments in laboratories (e.g. Ohnaka, 
2003). On the other hand, for the natural earthquake of $M 7$ class, it is on the order of $1 \mathrm{MJ} / \mathrm{m}^{2}$ (e.g., Beroza, and Spudich, 1988; Ide, 2003). This large difference of fracture energy, rather than stress, may be essential to explain wide scale difference and similarity of earthquake sources.

The difference of fracture energy is equivalent to the difference of slip-weakening distance, $D_{c}$, of the slipweakening friction law, if the stress state is uniform, which is a fairly feasible assumption compared to the variety of fracture energy. Assuming wide difference in slipweakening distance, Ide and Aochi (2005) have simulated dynamic rupture propagation on a planer fault. They prepared a heterogeneous distribution of $D_{c}$ using a random distribution of discrete orders of circular patches. Each patch has a uniform $D_{c}$, which is proportional to the patch size. Circular patches obey a power-law size-number relation, with a small number of large patches and many small patches. They successfully simulated many events of spontaneous rupture propagation and termination without special stopping mechanisms such as unbreakable barriers. They found some features similar to real earthquakes: (1) power law size-number statistics, (2) statistically selfsimilar rupture propagation, (3) subshear average rupture velocity with local super shear rupture velocity, and (4) socalled initial phases preceding the main moment release.

Despite of this success, there are still many things unsolved. One important problem is the relation between physically observable irregularity of a fault system and heterogeneity in slip-weakening distance and fracture energy. In this study, we propose a relation between fault topography and $D_{c}$ distribution. This relation makes continuous heterogeneity unlike discrete order patches in Ide and Aochi (2005). By changing parameters that characterize fault topography, we simulate dynamic rupture and calculate macroscopic parameters such as seismic moment, seismic energy, and rupture propagation velocity. We also investigate special cases with some characteristic lengths, as observable in real fault surfaces. To make the problem simple, we consider only 2D antiplane (mode III) and inplane (mode II) problems. From a numerous simulations of dynamic rupture propagation, we show that power-law size-number statistics is commonly observed for stopped events. However when the topography is not self-similar, most events break the whole model space and power-law statistics for stopped events does not guarantee the selfsimilarity of dynamic rupture propagation. Irregular topography sometimes accelerates or arrests rupture propagation, and such local changes of propagation velocity are important to calculate energy efficiency of earthquakes.

\section{Relation between Fault Topography and Slip- Weakening Distance}

When shear slip occurs between two contacting surfaces under high normal stress, asperities on surfaces suffer abrasion or other inelastic deformation before the frictional stress between these surfaces reaches a certain dynamic level. Assuming irregular surfaces and abrasion mechanism, Matsu'ura et al. (1992) showed that the characteristic displacement for the decrease of frictional stress, $D_{c}$, scales with the characteristic wavelength of surface irregularity,

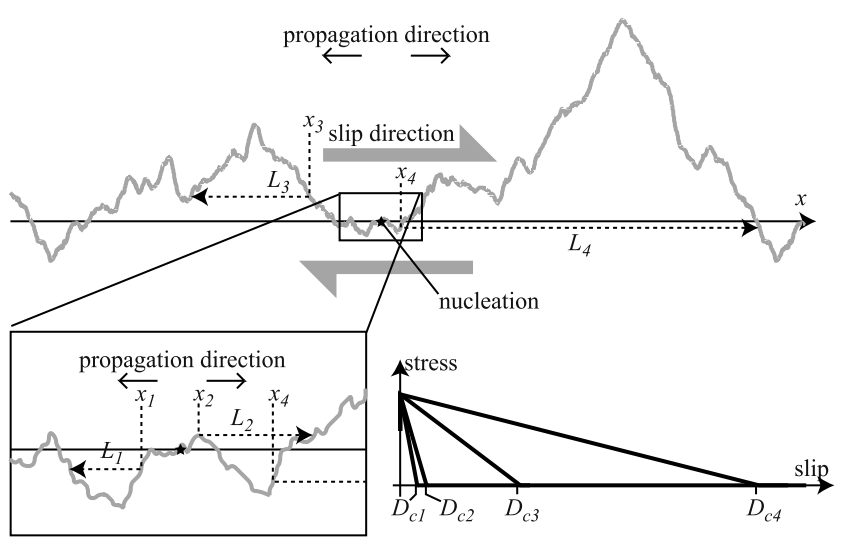

Fig. 1. The definition of irregularity size $L$ and slip-weakening distance $D_{c}$ in this study. We measure $L$ at any point on the fault surface, as the length to the first intersecting point between the surface and macroscopic rupture propagation direction (horizon) from the hypocenter (star). Examples of estimated $L$ at four points are shown. At each point on this surface, fault slip is governed by the slip-weakening friction law as shown with a slip-weakening distance $D_{c}$, which is proportional to the locally determined $L$.

Table 1. Parameter values.

\begin{tabular}{lll} 
Minimum boundary element length & $\Delta x_{0}$ & $1.0 \mathrm{~m}$ \\
Minimum time step (mode III) & $\Delta t_{0}$ & $0.5 \Delta x_{0} / \beta$ \\
(mode II) & & $0.5 \Delta x_{0} / \alpha$ \\
Yield stress & $\tau_{y}$ & $5 \mathrm{MPa}$ \\
Initial stress & $\tau_{0}$ & $3 \mathrm{MPa}$ \\
$P$-wave velocity & $\alpha$ & $6000 \mathrm{~m} / \mathrm{s}$ \\
$S$-wave velocity & $\beta$ & $3464 \mathrm{~m} / \mathrm{s}$ \\
Rigidity & $\mu$ & $30 \mathrm{GPa}$ \\
\hline
\end{tabular}

$\lambda_{c}$. The proportionality between $D_{c}$ and $\lambda_{c}$ has been confirmed by several laboratory experiments of rock friction and fracture as summarized by Ohnaka (2003). However, this relation is not directly introduced into numerical simulation to model heterogeneity of earthquake faults. If there is no characteristic length in surface topography, which is often observed for real faults and fracture surfaces, we cannot define $D_{c}$. Moreover, the relation between $\lambda_{c}$ and $D_{c}$ has been confirmed only macroscopically, while numerical simulation needs spatial distribution of frictional property. Therefore, we further assume that each point on the irregular fault has a local $D_{c}$, which is proportional to the irregularity size. This relation is not proved by Matsu'ura et al. (1992) or Ohnaka (2003), but we will see later that the macroscopic value of $D_{c}$ in Matsu'ura et al. (1992) and Ohnaka (2003) is considered as the appearance of the maximum $D_{c}$ when there is the limitation on the irregularity size.

This study uses a practical definition of irregularity size based on surface topography as illustrated in Fig. 1. The size is measured as the distance to the first intersecting point between the surface and macroscopic rupture propagation direction (horizon) in the direction of rupture propagation. Once the first rupture point, the hypocenter, is selected, we can measure the irregularity size, $L$, at any point on this surface. Slip-weakening distance $D_{c}$ is given as $D_{c}=$ $D_{c 0} \times L$, where $D_{c 0}$ is the proportionality constant. In 
(a) $\gamma=2, \delta=1.5$

(b) $\gamma=2.5, \delta=1.25$

(c) $\gamma=3, \delta=1.0$
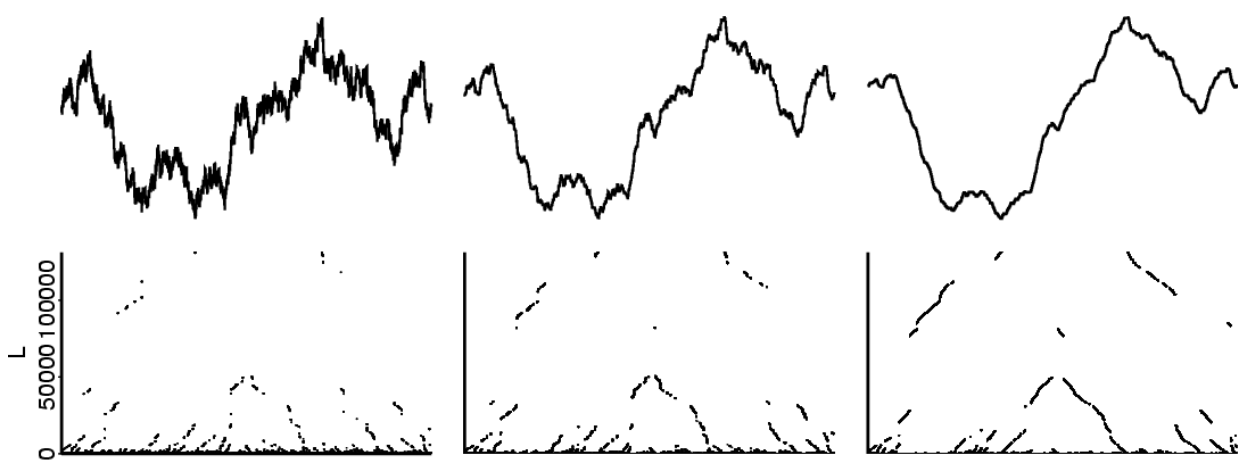

(d) $\gamma=3, \delta=1.0, \lambda_{L}=13107$

(e) $\gamma=3, \delta=1.0, \lambda_{\mathrm{S}}=131$
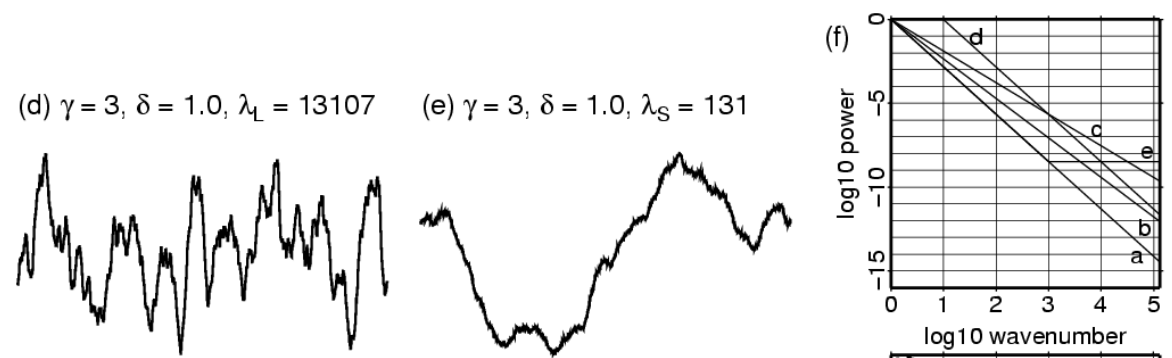

$\log 10$ wavenumber
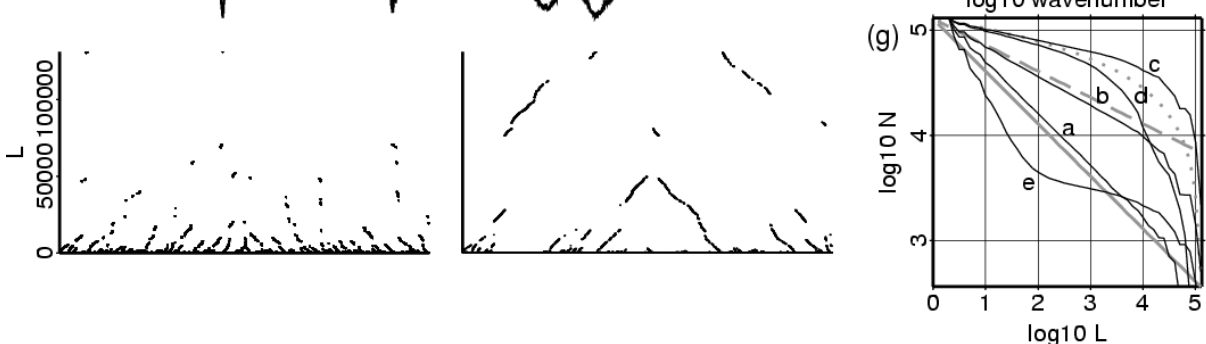

Fig. 2. (a)-(e) (top) Examples of topography (top) and distribution of irregularity size, $L$, (bottom) for different fractal dimensions $\delta$ and characteristic wavelengths $\lambda_{L}$ and $\lambda_{S}$, if any. (f) Power spectra of topography (a)-(e). (g) The number of point on the trace $N(L)$ where irregularity size is larger than $L$ (cumulative number). Five solid lines correspond to the examples (a)-(e). Gray solid, dashed, and dotted lines are proportional to $L^{-0.5}$, $L^{-0.25}$, and $\log \left(L_{\max } / L\right)$, respectively.

other words, $D_{c 0}$ is a normalized slip-weakening distance, determined by inelastic properties of surrounding material. When we assume a slip-weakening friction law, in which frictional stress $\tau_{f}$ is written as,

$$
\tau_{f}(\Delta u)=\left\{\begin{array}{ll}
\tau_{y}\left(1-\left(\Delta u / D_{c}\right)\right) & \Delta u<D_{c} \\
0 & \Delta u \geq D_{c}
\end{array},\right.
$$

the critical size of a stable $2 \mathrm{D}$ crack is given by $1.158 \mu^{*} D_{c} / \tau_{y}$ where $\mu^{*}=\mu$ (shear modulus) for mode III and $\mu /(1-v)$ for mode II, with $v$ being Poisson's ratio (Uenishi and Rice, 2003). Since a propagation crack must be larger than this size, the size $L$ is larger than $1.158 \mu^{*} D_{c 0} L / \tau_{y}$. Therefore, $D_{c 0}$ is on the order of $\tau_{y} / \mu^{*}$, which is $1.67 \times 10^{-4}$ with $\tau_{y}$ and $\mu$ of $5 \mathrm{MPa}$ and $30 \mathrm{GPa}$, respectively, which we assume in this study (Table 1).

We consider various types of fault surface topography each of which is characterized by a power spectrum and random phase in the wavenumber domain. Figure 2 shows examples of topography and corresponding distributions of local irregularity size determined based on the above definition and used in the following simulations. It should be noted that these distributions have some regularity as a natural result of our assumption. The irregularity size has a local maximum at every convex or concave peak of topography, and irregularity size is shorter than this value within the corresponding distance. We will discuss the effect of this regularity later. Figures 2(a), 2(b), and 2(c) show examples of topography that have power spectra of power law with the exponents $\gamma$ of $2.0,2.5$, and 3.0, respectively. There is no characteristic length except for the interval of grid points and size of model space. The relation between the exponent $\gamma$ and the fractal dimension of a self-affine fractal $\delta$, $\delta=(5-\gamma) / 2$, indicates that these examples have the fractal dimensions of $1.5,1.25$, and 1.0 , respectively.

While a power-law spectrum without characteristic size is statistically suggested by many previous studies in a wide scale range, individual spectra of topography usually have some characteristic sizes. The characteristic size in lowfrequency wavelength, a fractal limit for long size, determines the slip-weakening distance as shown by numerical model (Matsu'ura et al., 1992) and rock experiments (Ohnaka, 2003). Figure 2(d) show an example of topography that has a fractal limit $\lambda_{L}$, where no longer irregularity than $\lambda_{L}$ exists. In this case, $\lambda_{L}$ determines the maximum size of $L$ and hence the maximum $D_{c}$. We also consider a limit for short length, $\lambda_{S}$. In the field observation of Sagy et al. (2007), power spectra of topography measured along exhumed faults approaches to the slope of white noises in the short wavelength limit. Figure 2(e) is an example of 
this type, where the amplitude is flat at shorter wavelength than $\lambda_{S}$. In this case, the difference is hard to recognize visually because of the small amplitude of short-wavelength irregularity.

Figures 2(f) and 2(g) summarize the spectral shapes and the size-frequency statistics of irregularity size for the examples shown in Figs. 2(a)-(e). The relation between irregularity size $L$ and its cumulative number is approximated either by a power law, $L^{1-\delta}(\delta \neq 1)$, or by a logarithmic decay, $\log \left(L_{\max } / L\right)$, where $L_{\max }$ is the total model size. Therefore, density of the number of irregularity of size $L, d N(L)$, is proportional to $L^{-\delta}$. Based on fractal topography calculated by $\delta, \lambda_{L}, \lambda_{S}$, and randomly generated phase in the wavenumber domain, we obtain distribution of irregularity size, which is multiplied by $D_{c 0}$ to generate the distribution of slip-weakening distance $D_{c}$. For each combination of $\delta, \lambda_{L}$, and $\lambda_{S}$, we prepare 10 samples of topography for statistical discussion.

\section{Calculation of 2D Dynamic Rupture Propaga- tion}

Although we assumed irregular surface topography, this is not exactly used in the following calculation. It is theoretically possible to exactly calculate dynamic rupture propagation on highly heterogeneous interface including large change of normal stress as demonstrated by Aochi and Fukuyama (2002), but it costs too much and statistical investigation with a large number of case studies is practically impossible. In this study, we neglect surface geometry and normal stress change, and consider heterogeneity only in the distribution of slip-weakening distance proposed in the previous section. Fault is a line in a 2D infinite medium, on which slip occurs in inplane (mode II) or antiplane (mode III) direction.

To simulate realistic earthquakes that range at least from $M 0$ to $M 8$, we have to model approximately 4 order difference in the length scale. Therefore the total model space should have about $10^{5}$ discrete elements. Moreover statistical investigation requires numerous examples and thus fast computation. As an efficient method for this purpose, we adopt the renormalized boundary integral method (BIM) developed by Aochi and Ide (2004), in which renormalization connect slip distributions on two different scales. While Aochi and Ide (2004) and Ide and Aochi (2005) demonstrated the efficiency of the method in $3 \mathrm{D}$, this study uses a rather simple $2 \mathrm{D}$ version with the BIM representation of Cochard and Madariaga (1994) and Tada (1996). A unit model space consists of 64 boundary elements in space. For the $i$-th element in space and the $j$-th in time, the stress $T^{i j}$ and the slip rate $V^{i j}$ are calculated solving the discretized elastodynamic equation,

$$
T^{i j}=P^{0000} V^{i j}+\sum_{k=0}^{j-1} \sum_{l} P^{i j k l} V^{k l},
$$

and the discretized version of the constitutive Eq. (1),

$$
T^{i j}=F\left(V^{i j}\right),
$$

$P^{i j k l}$ is a kernel matrix representing interaction between the boundary elements. When the slipped area reaches the edge of the unit model space, we renormalize the slip rate of $n$ th scale $\left(V^{i j}\right)^{n}$ to the values of the next scale $\left(V^{i j}\right)^{n+1}$ by averaging as,

$$
\begin{aligned}
\left(V^{i j}\right)^{n+1}= & \left(\left(V^{2 i-1,2 j-1}\right)^{n}+\left(V^{2 i, 2 j-1}\right)^{n}\right. \\
& \left.+\left(V^{2 i-1,2 j}\right)^{n}+\left(V^{2 i, 2 j}\right)^{n}\right) / 4
\end{aligned}
$$

Each renormalization doubles the model space maintaining the number of elements. Using 64 spatial elements and 12 nested scales, we prepare a wide model space, $64 \times 2^{11}=$ 131072 unit elements of the finest scale. We confirmed that different settings, for example more spatial elements and less nested scales, $128 \times 4^{5}$ unit elements, make similar results. While a wider model is slow, it can reduce numerical noises that arise during renormalization, which sometimes reactivates once stopped rupture propagation. However, such strange behavior does not occur frequently and do not affect the result in a statistical sense.

We assign physical dimensions and units, to make easy comparison to real phenomena. The values are summarized in Table 1. As noted above, the stress field, initial, yield, and residual stress are uniform everywhere except for the initial rupture area. To start spontaneous rupture, we introduce a finite length of 'nucleus' where the initial stress level is the residual level at the beginning of calculation. The location of the center of the nucleus, the hypocenter, is selected randomly on the fault and around the hypocenter (location $i=0$ ) the stresses of 7 elements are reduced. This artificially introduced nucleus has infinite rupture propagation speed within itself and this effect remains visible until the rupture grows 3-5 times of the initial size. Then, a dynamic rupture propagates and stops, leaving a heterogeneous stress distribution. Before the next calculation from the different location, we reset the stress to the initial homogeneous state. Therefore, this calculation includes no longterm stress loading process. In practice, we select 1000 starting points for each combination of topography and $D_{c 0}$. Since we use 10 different shapes, we have 10000 realization of dynamic rupture in total for each set of $\delta, \lambda_{L}, \lambda_{S}$, and $D_{c 0}$.

Figure 3 represents an example of dynamic rupture propagation (event) on a 2D antiplane fault. These are spatiotemporal distributions of slip-rate on all scales. The rupture propagates from a nucleus satisfying the slip-weakening friction law with heterogeneous $D_{c}$. The slip-rate is renormalized to the next scale when the rupture front reaches the edge of the model space. After 8 iterations of renormalizations, this event eventually stops on the ninth scale. We calculate several macroscopic parameters that characterize dynamic behavior of each event. The seismic moment is the integration of the slip-rate $V^{i j}$ with time and along the fault, multiplied by the rigidity, $\mu$,

$$
M_{0}=\mu \Delta x \Delta t \sum_{i j} V^{i j},
$$

where $\Delta x$ and $\Delta t$ are the spatial and the temporal sizes of boundary element, respectively. While the seismic moment is measured on each scale during the growth of one event using (5), we use the final value of the seismic moment 

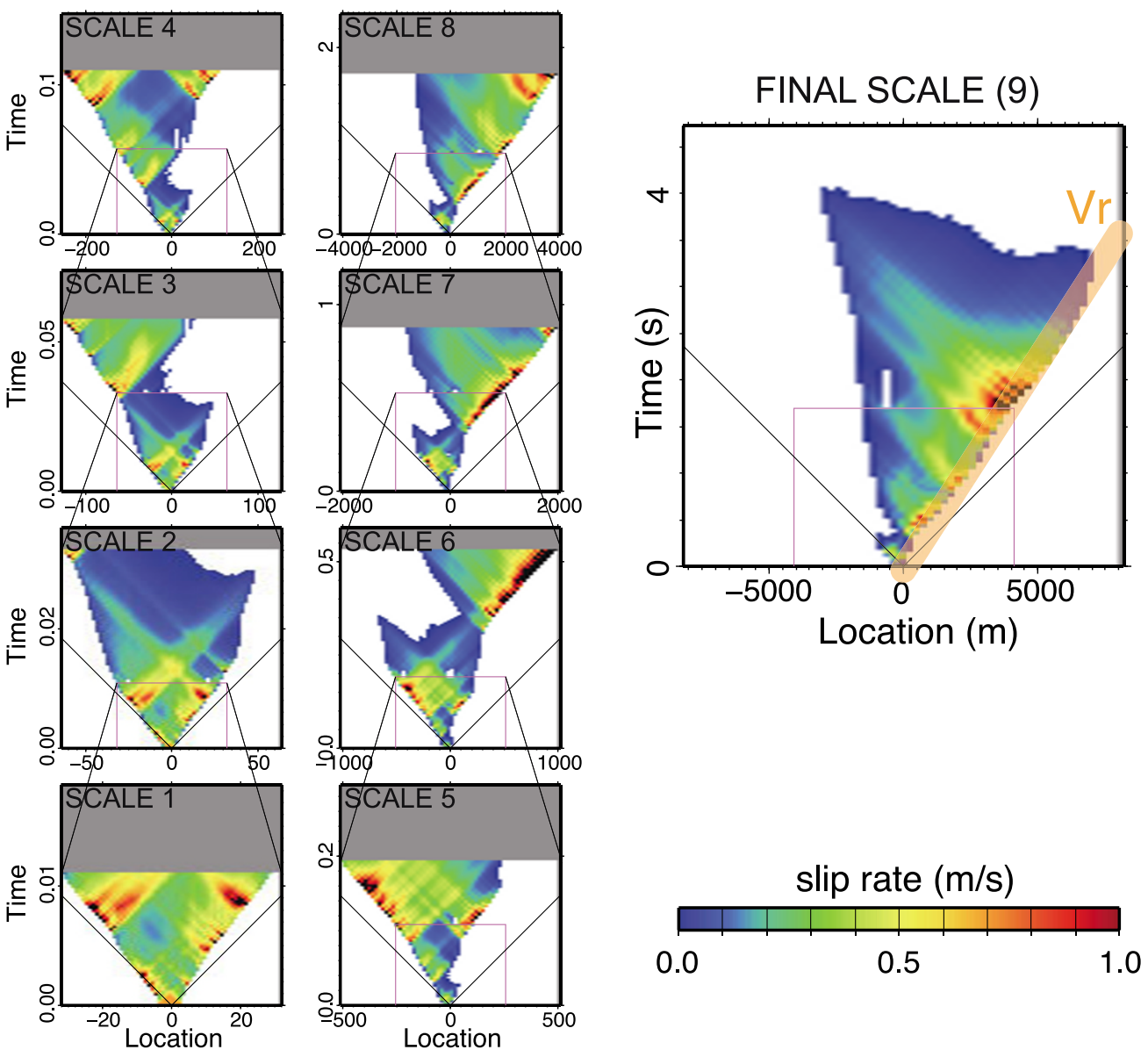

Fig. 3. An example of dynamic rupture of mode III. Each panel (scale 1-8) represents spatio-temporal distribution of slip rate on that scale till the rupture front reaches one edge of the model space. Each distribution is renormalized to the small part of the distribution on the next scale as shown by a purple square. The largest panel shows slip-rate distribution in the final scale (scale 9) till the end of whole calculation. Calculated average rupture velocity $V_{r}$ is shown by an orange bold line.

when we discuss size-number statistics. The moment magnitude corresponds to the seismic moment as,

$$
M_{\mathrm{w}}=\log M_{0}+C,
$$

where the constant $C$ is selected to set the moment magnitude of an initial nucleus as 0 . Because we consider 2D faults, the coefficient before the $\log$ moment is 1 , instead of 1.5 for $3 \mathrm{D}$ natural earthquakes. Our nucleus size is $7 \mathrm{~m}$ and it is comparable to the typical size of $M 0$ natural earthquake of about $10 \mathrm{~m}$. We discuss the rupture propagation velocity as the average of rupture propagation velocities from a fixed start point, the hypocenter, weighted by the values of slip rate. Practically, it is given as,

$$
V_{r}=\frac{\sum_{i j}\left|V^{i j}([i]-0.5)\right|^{2}}{\sum_{i j}\left|V^{i j}\right|^{2}([i]-0.5)(j+0.5)} \frac{\Delta x}{\Delta t} .
$$

The summation is taken for all boundary elements. It should be noted that this value represents average velocity of rupture propagation and local propagation velocity can be much faster or slower.

The seismic energy $E_{s}$ and the total fracture energy $E_{G}$ are useful parameters to compare with real earthquakes. They are calculated using the expression of Kostrov (1974), with slip $\Delta u(x, t)$ and stress $\tau(x, t)$ along the fault,

$$
E_{s}=\frac{1}{2} \int_{-\infty}^{\infty}(\tau(x, 0)-\tau(x, \infty)) \Delta u(\mathbf{x}, \infty) d x-E_{G},
$$

$$
E_{G}=-\int_{0}^{\infty} \int_{-\infty}^{\infty}\left(\frac{\partial}{\partial t} \tau(x, t)\right) \Delta u(x, t) d x d t
$$

These equations are discretized as demonstrated by Ide $(2002,2003)$ and written at the end of calculation (time $j$ ) as

$$
E_{s}=\Delta t \Delta x \sum_{i}\left[\sum_{k=0}^{j} V^{i k}\right]\left(T^{i 0}-T^{i j}\right) / 2-E_{G},
$$

$$
E_{G}=\Delta t \Delta x \sum_{i} \sum_{l=1}^{j}\left(2 \sum_{k=0}^{l-1} V^{i k}+V^{i l}\right)\left(T^{i, l-1}-T^{i l}\right) / 2 .
$$

Figure 4 graphically shows the amounts of the seismic energy and the fracture energy calculated using these equations. 


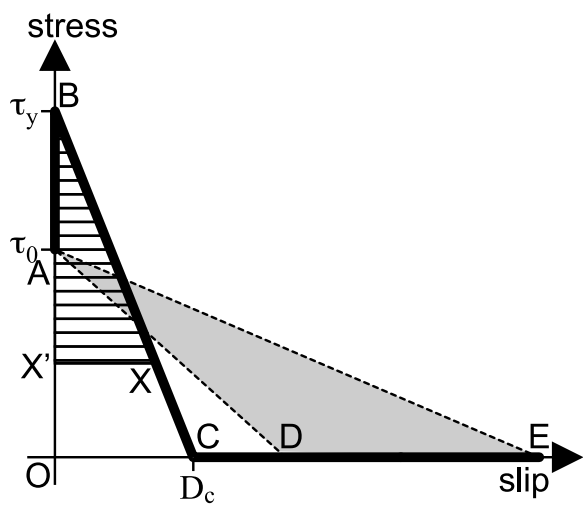

Fig. 4. Slip-stress diagram to show the locally calculated fracture energy and the contribution to seismic energy. Stress changes with slip along A-B-C-D-E. Fracture energy is estimated locally at any time, while seismic energy is measured after the rupture termination by integration of local contribution across fault area. When slip is less than $D_{c}$, at point $\mathrm{X}$, fracture energy is given by the hatched area $\mathrm{BXX}$ and the negative contribution to seismic energy is the area $\mathrm{ABX}$. When slip exceed $D_{c}$, at point $\mathrm{E}$, fracture energy is $\mathrm{BOC}(=\mathrm{AOD})$ and the contribution to seismic energy is ADE.

\section{Statistical Properties of Rupture Events \\ 4.1 Topography without characteristic length}

Using the macroscopic parameters defined in the previous section, we study the statistical features of dynamic rupture for topography without characteristic length as shown in Figs. 2(a)-(c).

Figures 5(a) and (b) show the number of events of magnitude $M$ and those of magnitude larger than $M$ (cumulative number), respectively, for mode III rupture with combinations of three fractal dimension $\delta$ and three normalized slip-weakening distance $D_{c 0}$. The cumulative number decreases as the rupture grows, but remains constant above some size especially for $\delta=1.25$ and 1.5. Large population of large events of $M 8$ means that there are significant numbers of events that do not stop spontaneously and break the whole model space. We refer to this type of events as nonstopping events and distinguish them from spontaneously stopped events. Relative ratio between large and small events depends on $D_{c 0}$. A large $D_{c 0}$ yields large average slip-weakening distance and large fracture energy, which effectively stop rupture propagation and reduce the probability of large events. When $D_{c 0}$ is larger than $6 \times 10^{-4}$, no rupture propagation occurs because the size of initial nucleus is much smaller than the critical length.

When $\delta=1$, most events stop spontaneously and the cumulative size-number relation approximately obeys a power law with a steep slope for a large $D_{c 0}$. This behavior is clearer in the non-cumulative plot (Fig. 5(a)) where we can exclude the contribution of initial nucleus smaller than $M 1$ and non-stopping events larger than $M 7$. Except for these small and large events, all stopped events from $M 1$ to $M 7$ seem to obey a power law size-number statistics even for $\delta=1.25$ and 1.5 .

Mode II events behave almost similarly to the mode III ones. Figures 5(c) and 5(d) show the results for mode II, where only the direction of slip is different from the configuration of mode III shown in Figs. 5(a) and 5(b). The major difference is the value of $D_{c 0}$. Since the crack exten-
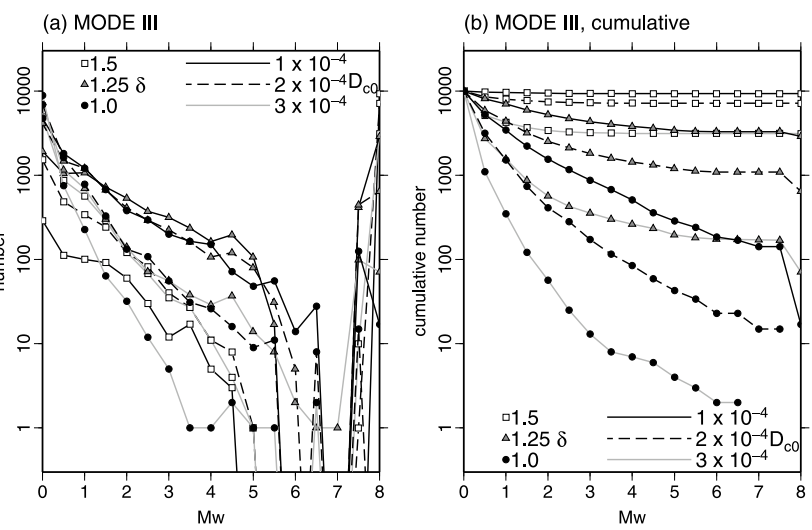

(c) MODE II
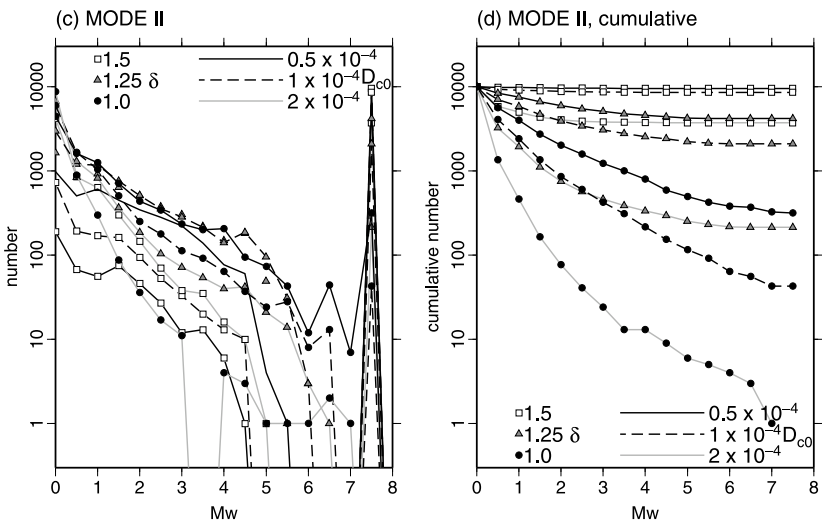

Fig. 5. Size (moment magnitude) and number statistics of events for various combinations of $\delta$ and $D_{c 0}$. (a) Number of events from $M_{\mathrm{w}}$ to $M_{\mathrm{W}}+0.5$ for mode III. (b) Cumulative number of events larger than $M_{\mathrm{w}}$ for mode III. (c) Number of events from $M_{\mathrm{w}}$ to $M_{\mathrm{w}}+0.5$ for mode II. (d) Cumulative number of events larger than $M_{\mathrm{w}}$ for mode II. Circle, triangle, and square symbols represent the fractal dimension $\delta$ of 1.5 , 1.25 , and 1.0, respectively. Solid black, dashed black, and gray lines connect the values for different values of the normalized slip-weakening distance $D_{c 0}$ as shown in each panel.

sion force of a mode II crack is smaller than that of mode III at a slower rupture velocity (e.g., Freund, 1998), smaller fracture energy is required to stop rupture propagation.

The linear relation between magnitude and log number suggests the self-similarity of rupture as Ide and Aochi (2005) demonstrated. To check it, we monitor the change of rupture propagation velocity during an event growth. If the self-similarity holds, the rupture looks similarly at any moments of rupture propagation and the rupture velocity is constant. Figure 6(a) summarizes the magnitude and rupture velocity at every scale for all events in mode III calculation. We neglect values in the smallest scale, because the effect of initial nucleus is dominant and the estimated rupture velocity is quite high. Except for these small events, rupture propagation velocity changes almost monotonically. When the fractal dimension $\delta$ is 1 , the velocity is almost constant, while it is accelerated to the terminal velocity, the $S$ wave velocity, for $\delta \neq 1$. We can conclude that the rupture propagates statistically self-similarly only when $\delta=1$, namely on a self-similar fault surface. Although the stopped events usually show power-law size-number statistics, the rupture growth for $\delta \neq 1$ is not self-similar. Since a normalized slip-weakening distance $D_{c 0}$ is proportional to the average fracture energy, large $D_{c 0}$ results in slow rupture in average. 
(a) MODE III

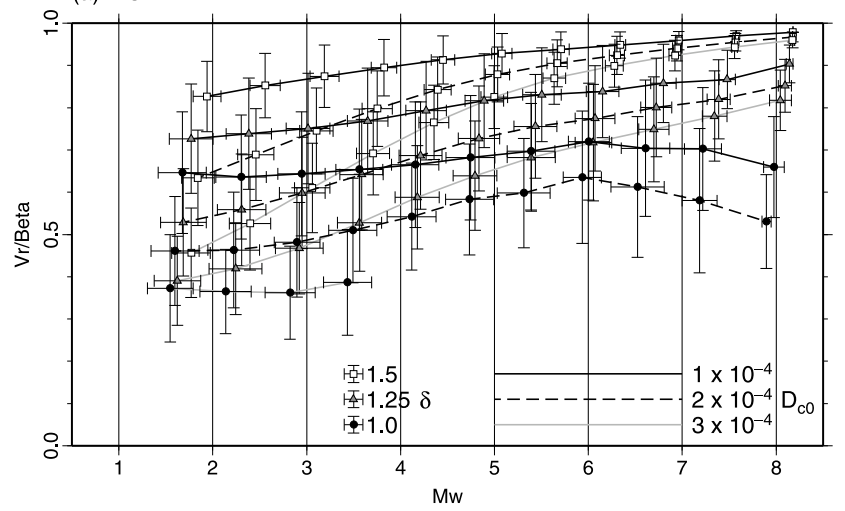

(b) MODE II

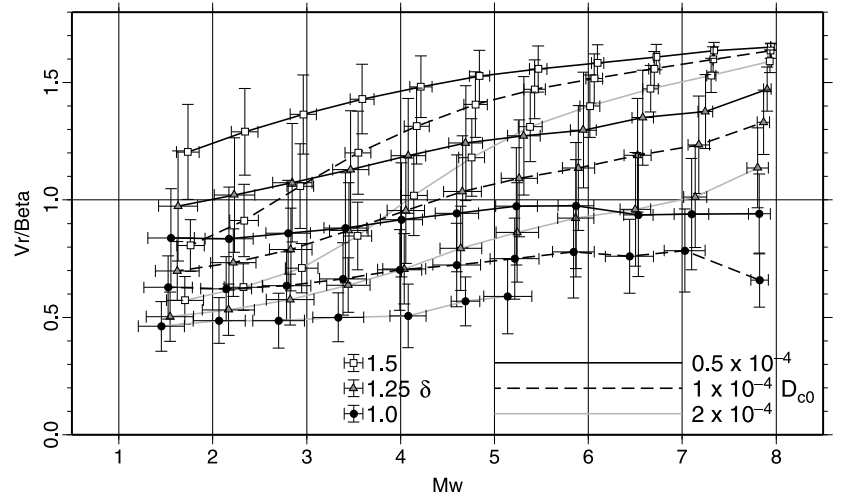

Fig. 6. Comparison between moment magnitude $M_{\mathrm{W}}$ and rupture velocity $V_{r}$ measured for each scale, from the second to the 12th scales. We neglect the first scale. Each symbol shows the average value at the end of calculation on the scale with an error bar showing the standard deviation. Circle, triangle, and square symbols represent the fractal dimension $\delta$ of 1.0, 1.25, and 1.5, respectively. Solid black, dashed black, and gray lines connect the values for the different normalized slip-weakening distance $D_{c 0}$ as shown in the bottom of each panel. (a) Mode III (antiplane) calculation, where the terminal velocity is shear wave velocity. (b) Mode II (inplane) calculation, where the terminal velocity is 1.73 of shear wave velocity.

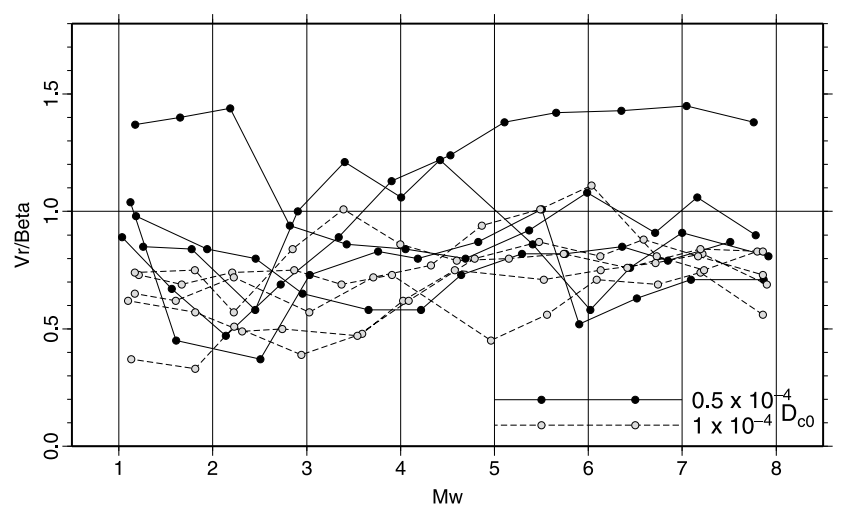

Fig. 7. Circles connected by a line show the change of rupture velocity during a non-stopping event of mode II rupture. This is calculated for $\delta=1$, and $D_{c 0}=0.5 \times 10^{-4}$ (black circles with solid line) and $1 \times 10^{-4}$ (gray circles with dashed line). Five events are randomly selected for each $D_{c 0}$.

Figure 6(b) shows the result for mode II crack, where super shear rupture propagation is possible, and terminal velocity is the $P$ wave velocity as shown by Andrews (1976). However, the rupture propagation velocity remains subshear for (a) MODE III

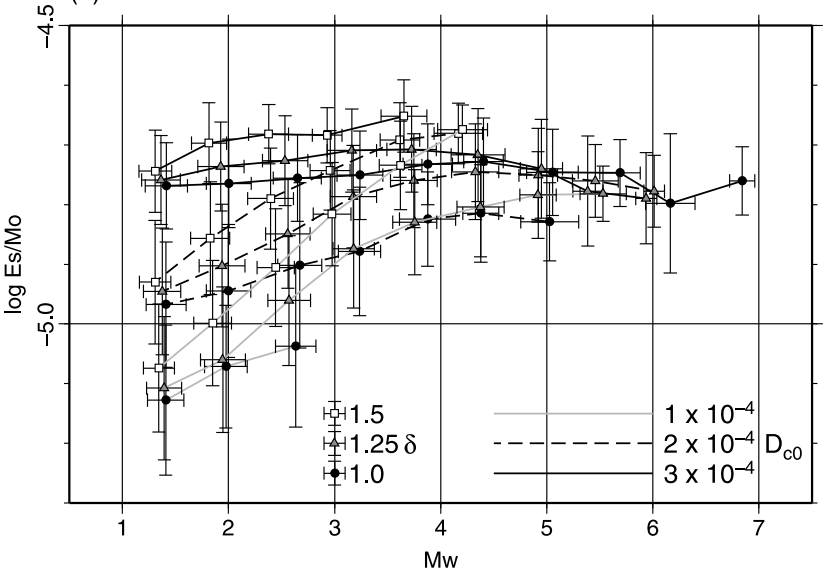

(b) MODE II

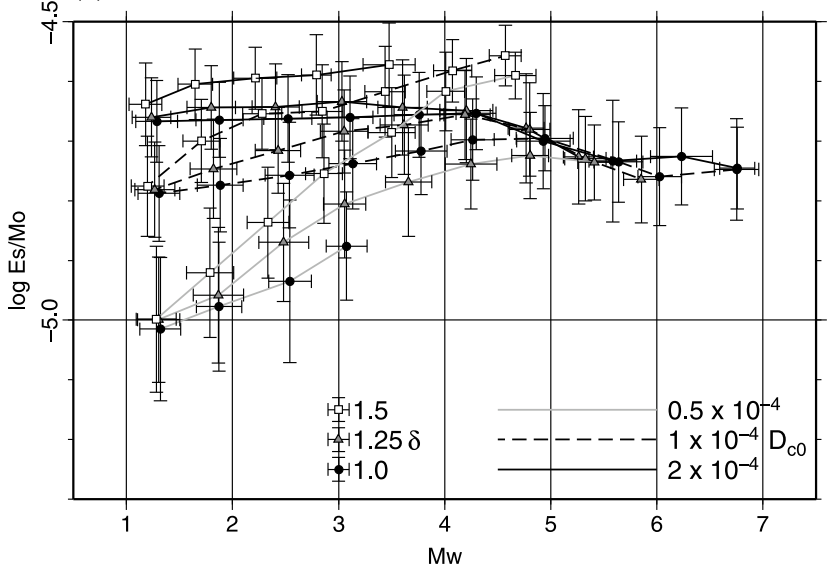

Fig. 8. Moment magnitude and scaled energy, the ratio between seismic energy and seismic moment, of all stopped events except for those stopped on the first scale. (a) Mode III and (b) mode II. Each symbol shows the average value at the end of calculation on the scale with an error bar showing standard deviation. Circle, triangle, and square symbols represent the fractal dimension $\delta$ of $1.0,1.25$, and 1.5 , respectively. Solid black, dashed black, and gray lines connect the values for the different normalized slip-weakening distance $D_{c 0}$ as shown in the bottom of each panel.

self-similar rupture with $\delta=1$.

Even under a selfsimilar condition with $\delta=1$, rupture often propagates at a local propagation velocity much faster than the average value. Figure 7 shows magnitude and rupture velocity during 10 randomly selected non-stopping events in mode II. Each rupture experiences different rupture velocity at different size. Nine out of ten events in Fig. 7 propagate at a subshear rupture velocity in the final scale, but some of them experience super shear propagation during the growth. Two events propagate at the velocity of about $\sqrt{2} \beta$, which is the velocity at which super shear rupture can propagate stably.

Figure 8 shows the ratio of seismic energy to seismic moment (scaled energy) for nine combinations of $\delta$ and $D_{c 0}$. Averages and standard deviations are calculated for log scaled energy. Since seismic energy can be measured after the termination of the rupture, this figure includes only stopped events, all except for the events that stopped on the first scale. When a ruptured area is small, the seismic energy is determined mainly by $D_{c 0}$, because the rupture is stopped by small scale heterogeneity almost irrelevantly to 


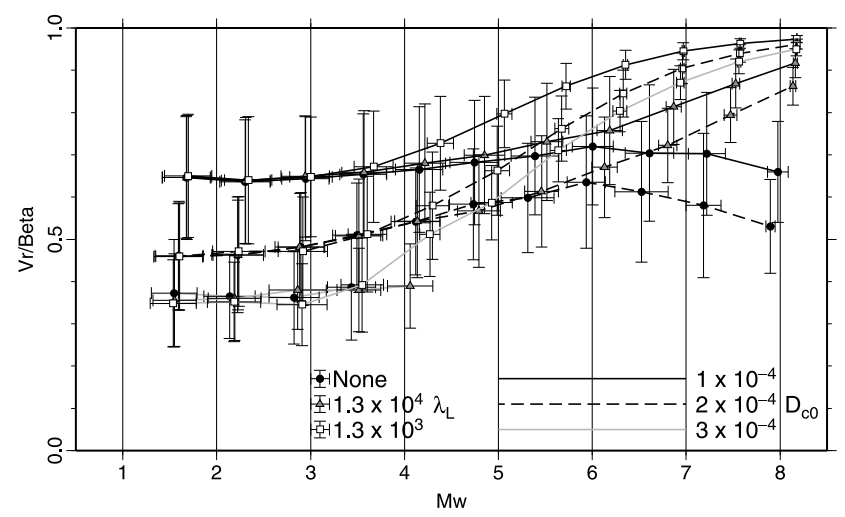

Fig. 9. Comparison between moment magnitude $M_{\mathrm{w}}$ and rupture velocity $V_{r}$ measured for each scale, from the second to the 12 th scale, calculated for the case with $\lambda_{L}$. We neglect the first scale. Each symbol shows the average value at the end of calculation on the scale with an error bar showing the standard deviation. Circle, triangle, and square symbols represent results using $\lambda_{L}$ of infinity (no limit), $1.3 \times 10^{4} \mathrm{~m}$, and $1.3 \times 10^{3} \mathrm{~m}$, respectively. Solid black, dashed black, and gray lines connect the values for the different normalized slip-weakening distance $D_{c 0}$ as shown in the bottom of each panel.

large scale fractal structure and $\delta$. The difference between Figs. 8(a) and 8(b) arise mainly from the difference of $D_{c 0}$, rather than due to the difference of rupture modes. Topography with larger $\delta$ tends to produce energetic events as rupture grows. It is not surprising that scaled energy is constant for the case $\delta=1$ because rupture propagates self-similarly at any moment. Moreover, even for non-self-similar cases of $\delta \neq 1$, the scaled energy looks constant if $D_{c 0}$ is small. This suggests that the self-similarity of rupture process is not guaranteed only by the constant scaled energy, which is often observed for natural earthquakes.

\subsection{Topography with characteristic length}

Next we consider the situation where topography has a characteristic length that defines a longer or shorter limit of power law regime in spectrum. Since mode II and III give essentially the same behavior in the previous section, we focus on the simple mode III problem and fix $\delta$ to 1 . Therefore rupture propagates statistically self-similarly if there is no characteristic length.

Figure 9 shows the relation between rupture propagation velocity and seismic moment when topography has longer limit of fractal, $\lambda_{L}$. As shown in Fig. 2(d), such topography has no irregularity larger than $\lambda_{L}$. Therefore, once a rupture grows beyond a characteristic size, it is accelerated to the terminal velocity and break whole model space. This is a well-known behavior of traditional dynamic rupture simulations with a constant $D_{c}$. The characteristic size is about $M_{\mathrm{w}} 4$ for $\lambda_{L}$ of $1.3 \times 10^{3} \mathrm{~m}$ and $M_{\mathrm{w}} 6$ for $\lambda_{L}$ of $1.3 \times 10^{4} \mathrm{~m}$. The actual acceleration starts from a little smaller size probably due to the effect of inertia. To stop such rupture, we have to introduce some special mechanisms such as unbreakable barriers and a wide unstressed area, which are often assumed in many previous studies of dynamic rupture propagation.

There can be a limit for shorter wavelength of irregularity $\lambda_{S}$, below which topography looks like white noise as shown in Fig. 2(e). Although the difference of topography (a)

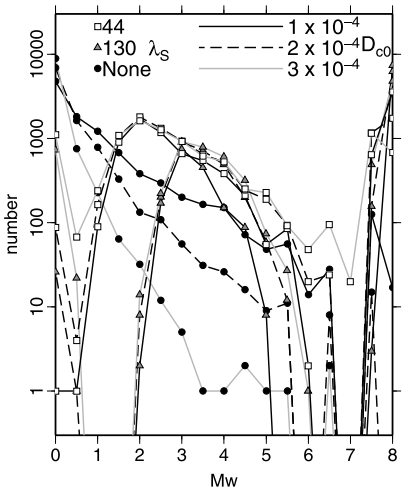

(b)

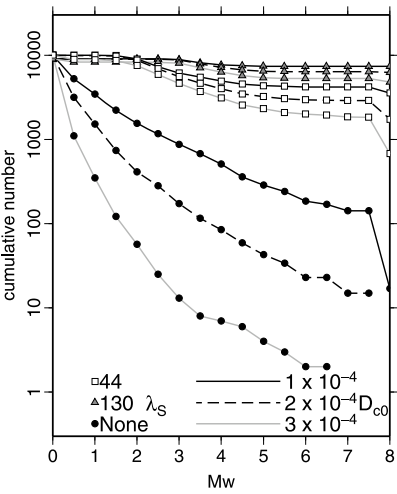

Fig. 10. Size (moment magnitude) and number statistics for topography with short wavelength limit $\lambda_{S}$. (a) Number of events from $M_{\mathrm{w}}$ to $M_{\mathrm{w}}+0.5$. (b) Cumulative number of events larger than $M_{\mathrm{w}}$. Circles show the relations without $\lambda_{S}$, for comparison. Squares and triangles represent $\lambda_{S}=44 \mathrm{~m}$ and $130 \mathrm{~m}$, respectively. Solid black, dashed black, and gray lines connect the values for the different normalized slip-weakening distance $D_{c 0}$ as shown in each panel.

is hard to see in larger scale, the average value of $D_{c}$ is small and the chance of rupture arrest is much smaller. Hence, most events are non-stopping event. Figure 10 shows sizenumber relation for $\lambda_{S}=44 \mathrm{~m}$ and $130 \mathrm{~m}$. Nevertheless we observe a peak in non-cumulative size-number relation (Fig. 10(a)) that rises from $M_{\mathrm{w}} \sim 1.3$ or 2.2 corresponding to either $\lambda_{S}$, and falls gradually. The rupture is best stopped at this size, which is a characteristic size of this system. Similar behavior is expected for any concave sections of topography spectra. Sagy et al. (2007) found similar concave section in the topography spectra in the slip direction of mature faults, which is known to have characteristic earthquakes in the magnitude-frequency distribution of seismicity (e.g., Stirling et al., 1996). The peaks in Fig. 10(a) may represent such characteristic earthquakes.

\section{Discussion}

\subsection{Radiation efficiency}

The radiation efficiency $\eta_{R}$ represents the efficiency of seismic energy radiation compared with the total release of potential energy minus frictional loss. It is calculated for each event in this study using the seismic energy $E_{s}$ and the fracture energy $E_{G}$ defined in Eqs. (8)-(11) as,

$$
\eta_{R}=E_{s} /\left(E_{s}+E_{G}\right) \text {. }
$$

The radiation efficiency of mode III crack propagating selfsimilarly at a constant rupture velocity $V_{R}$ is given as (e.g. Freund, 1998):

$$
\eta_{R}=1-\sqrt{\left(1-V_{R} / \beta\right) /\left(1+V_{R} / \beta\right)} .
$$

Figure 11 compares average rupture velocity and radiation efficiency for all the stopped events larger than $M_{\mathrm{w}} 1.5$. Generally $\eta_{R}$ is larger for fast ruptures as the analytic expression predicts, but the increase ratio is slower than predicted. This behavior is interpreted as follows. Slow ruptures tend to radiate more energy than the self-similar crack because they include small-scale fast ruptures that excite seismic energy more efficiently. On the other hand, fast 


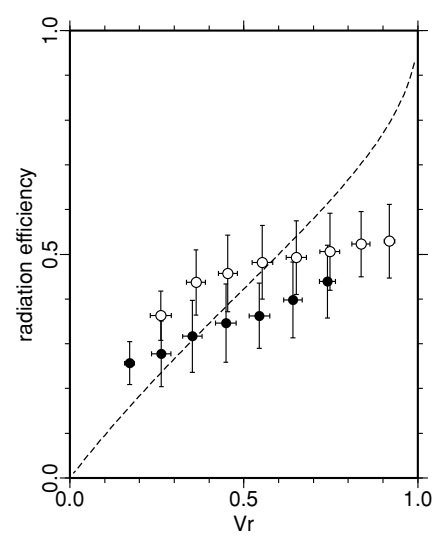

Fig. 11. Comparison between rupture velocity and radiation efficiency for stopped events larger than $M_{\mathrm{w}} 1.5$. A circle represents average values measured for each 0.1 bin of rupture velocity. Open and black circles are for $D_{c 0}=1 \times 10^{-4}$ and $2 \times 10^{-4}$, respectively. Error bars represent the standard deviation for each group. A dashed curve shows the analytic solution for a mode III self-similar crack propagating at a constant velocity.

ruptures radiate seismic energy efficiently during the rupture propagation. However they have to consume some fracture energy to be arrested, which reduces total seismic efficiency. This suggests that for any stopped earthquake, radiation efficiency cannot be as large as 80-90\% and fracture energy required to stop the rupture is comparable to seismic energy.

\subsection{Spatial distribution of $D_{c}$}

Although the distribution of slip-weakening distance $D_{c}$ is determined based on random topography, it has some regularity due to the definition of $D_{c}$ as shown in Fig. 2. This regularity is essential for the statistical self-similarity and power-law size-number relation described so far. If the spatial distribution of $D_{c}$ is random, namely if the original distribution is mixed up randomly in space, the behavior of the rupture propagation is quite different. Figure 12 shows size-number relations for several random $D_{c}$ distributions compared with the previously mentioned size-number relations of regular distribution. Random distributions yield less large events than relatively regular distributions, suggesting the regularity introduced in this study promotes rupture growth. No power-low section is observable for random distribution with $\delta=1$, but visible with $\delta=1.5$ below a limiting size.

The relations in Fig. 12 are explained analytically to some extent. As shown in Fig. 2(g), the size-number relations of $L$ has a density function $d N(L)$ proportional to the negative power of the fractal dimension $\delta$. We assume that the probability density function of $D_{c}, p(x)$, is proportional to $x^{-\delta}$, and for the case $\delta \neq 1$,

$$
p(x)=\frac{1-\delta}{\left(D_{\max }\right)^{1-\delta}-\left(D_{\min }\right)^{1-\delta}} x^{-\delta},
$$

where $D_{\max }$ and $D_{\min }$ are the maximum and the minimum value of $D_{c}$. Since the stress condition is homogeneous, fracture energy is proportional to $D_{c}$. Rupture propagation is arrested if fracture energy is larger than the energy release rate of the crack, which scales as the length $n \Delta x$. Therefore there is a critical value of $D_{c}$ above which the rupture is
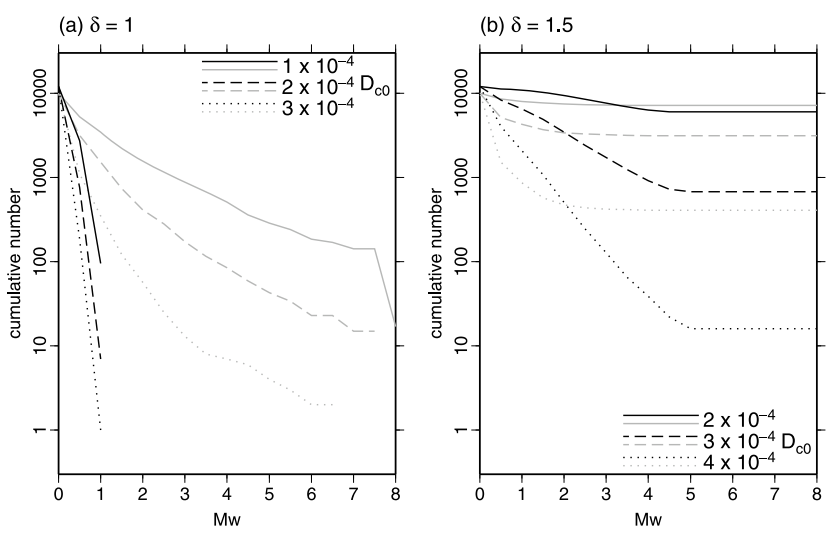

Fig. 12. Size (moment magnitude) and number statistics of rupture events to compare difference of spatial distribution of $D_{c}$. Cumulative numbers of events larger than $M_{\mathrm{w}}$ are shown for (a) $\delta=1$ and (b) $\delta=1.5$. Black and gray lines show results for random distributions and relatively regular distributions, respectively. Different types of line represent the different values of normalized slip-weakening distance $D_{c 0}$ as shown in each panel.

arrested, which is proportional to $n$ and we write $D_{\text {stop }}=$ $A n$. The probability that rupture propagation of length $n \Delta x$ is stopped at one end is

$$
\begin{aligned}
P_{n} & =\int_{D_{\text {stop }}}^{D_{\max }} p(x) d x=\frac{\left(D_{\max }\right)^{1-\delta}-(A n)^{1-\delta}}{\left(D_{\max }\right)^{1-\delta}-\left(D_{\min }\right)^{1-\delta}} \\
& \sim\left(A n / D_{\min }\right)^{1-\delta}-\left(D_{\max } / D_{\min }\right)^{1-\delta} .
\end{aligned}
$$

The above probability is valid only for small cracks, $n<$ $D_{\max } / A$ and $P_{n}=0$ for $n>D_{\max } / A$, which means that rupture cannot be arrested above this size. When there are $N_{n}$ events of length $n \Delta x$, the number of events of length $(n+1) \Delta x, N_{n+1}$ is, considering both ends,

$$
N_{n+1}=N_{n}\left(1-\left(P_{n}\right)^{2}\right)=N_{1} \prod_{i=1}^{n}\left(1-\left(P_{i}\right)^{2}\right) .
$$

When $\delta=1.5$, and $1 \ll n \ll D_{\max } / A$,

$$
\begin{aligned}
\log N_{n} & \sim \log N_{1}+\sum_{i=1}^{n-1} \log \left(1-\frac{D_{\min }}{A i}\right) \\
& \sim-\frac{D_{\text {min }}}{A} \log n+\text { const. }
\end{aligned}
$$

Since $\log n$ is proportional to the magnitude, $\log N$ decreases linearly as $M_{\mathrm{w}}$ increases. When $n$ exceeds $D_{\max } / A$, the slope becomes flat, indicating there are no stopped event above this size.

This simple calculation shows that not only size-number distribution of slip-weakening distance is important to consider rupture behavior on a heterogeneous fault plane. Definition of $D_{c}$ in Fig. 1 introduces regularity into the spatial distribution of $D_{c}$. With this definition, once a rupture breaks an element of large $D_{c}$, it can propagate at least the size of the corresponding irregularity. This mechanism promotes rupture propagation and provides completely different size-frequency statistics from that in a spatially random $D_{c}$ model. 


\section{Conclusion}

Earthquakes are dynamic ruptures starting from very small area that has not been identified from seismic observation. They propagate radiating seismic energy within complex media with various size of heterogeneity. To realize statistical representation of such processes, we simulate dynamic propagation of rupture on a plane where fracture energy is distributed heterogeneously. To keep problems simple, we consider 2D in-plane (mode II) and antiplane (mode III) problems with a homogeneous stress condition. Nevertheless, the rupture behavior is diverse because of the heterogeneous distribution of fracture energy, or slip-weakening distance $D_{c}$, which is assumed based on the fractal topography of fault plane. We define $D_{c}$ as a value that is proportional to the locally measured size of topographic irregularity. Therefore we have two parameters that characterize distribution of $D_{c}$, the fractal dimension of topography $\delta$ and the normalized slip-weakening distance $D_{c 0}$. We also consider the maximum and the minimum fractal limits of this distribution as special cases. Carrying out a large number of dynamic rupture simulations, we discuss the size-number statistics of events and the scaling relations between macroscopic parameters such as seismic moment, seismic energy, fracture energy, and average rupture propagation velocity.

Most ruptures spontaneously stop and the size-number statistics of these events is approximately power law when $\delta=1$, for $D_{c}$ distribution based on self-similar topography. The exponent of power depends on normalized slipweakening distance $D_{c 0}$, and a smaller $D_{c 0}$ results in many large events because the average fracture energy is small. $D_{c 0}$ also controls the average rupture velocity, which is statistically scale invariant. During rupture propagation, rupture velocity is variable and sometimes locally exceeds the $S$-wave velocity in mode II rupture. These are similar characteristics observed in the previous dynamic models using circular patches by Ide and Aochi (2005). When the fractal dimension $\delta>1$, most events do not stop and are accelerated to the terminal rupture propagation velocity. However, even for $\delta>1$, the size-number statistics of stopped events is approximately a power law. These stopped events for $\delta>1$ show size dependence of the ratio of seismic energy to seismic moment, scaled energy, while the dependence is weak when $\delta=1$. This suggests that we may observe different scaling of scaled energy when scale-dependence of irregularity is different.

If there is the upper fractal limit of wavelength in fractal topography, there is no large irregularity that can stop propagating rupture above this limit and all events become unstopping events, which is unlikely in natural earthquake environment. On the other hand, a lower limit determines a characteristic size of event in size-number statistics, which may be correspond to characteristic earthquakes observed in some mature fault systems. The power spectrum of topography of a natural fault has some concave sections, which may act like a fractal limit and make characteristic behavior of earthquakes.

The rule to determine $D_{c}$ distribution adopted in this study is just a candidate that connects natural fault system and $D_{c}$ distribution. Although this uses a continuous topog- raphy, it is not a typical appearance of natural fault system and we need to consider other complexity such as branches and steps in future. Nevertheless, as we show in the discussion, the regularity of spatial distribution of $D_{c}$ is a key to consider rupture in heterogeneous media and this may hold for other kind of heterogeneity, too. Characteristics such as $\delta$-dependent scaling of scaled energy and relatively small radiation efficiency can be explained by a fundamental energy balance and will persist in more complex simulations.

Acknowledgments. I thank to the comments from two anonymous referees. This work is supported by the DaiDaiToku Project, Special Project for Earthquake Disaster Mitigation in Metropolitan Tokyo Area, and Grant-in-Aid for Scientific Research, MEXT, Japan.

\section{References}

Andrews, D. J., Rupture velocity of plane strain shear cracks, J. Geophys. Res., 81, 5679-5687, 1976.

Aochi, H. and E. Fukuyama, Three-dimensional nonplanar simulation of the 1992 Landers earthquake, J. Geophys. Res., 107, doi:10.1029/ 2000JB000061, 2002.

Aochi, H. and S. Ide, Numerical study on multi-scaling earthquake rupture, Geophys. Res. Lett., 31, doi:10.1029/2003GL018708, 2004.

Beroza, G. and P. Spudich, Linearized inversion for fault rupture behavior: Application to the 1984 Morgan Hill, California, earthquake, J. Geophys. Res., 93, 6275-6296, 1988.

Brown, S. R. and C. H. Scholz, Broad bandwidth study of the topography of natural rock surfaces, J. Geophys. Res., 90, 12,575-12,582, 1985.

Cochard, A. and R. Madariaga, Dynamic faulting under Rate-dependent friction, PAGEOPH, 142, 419-445, 1994.

Day, S., Three dimensional simulation of spontaneous rupture: the effect of nonuniform prestress, Bull. Seismol. Soc. Am., 72, 1881-1902, 1982.

Freund, L. B., Dynamic Fracture Mechanics, Cambridge Univ. Press, New York, 1998.

Guatteri, M., P. M. Mai, G. C. Beroza, and J. Boatwright, Strong-ground motion prediction from stochastic-dynamic source models, Bull. Seismol. Soc. Am., 93, 301-313, 2003.

Ide, S., Estimation of Radiated energy of finite-source earthquake models, Bull. Seismol. Soc. Am., 92, 2294-3005, 2002.

Ide, S., On fracture surface energy of natural earthquakes from viewpoint of seismic observations, Bull. Earthquake Res. Inst., 78, 1-120, 2003.

Ide, S. and H. Aochi, Earthquakes as multiscale dynamic rupture with heterogeneous fracture surface energy, J. Geophys. Res., 110, doi:10. 1029/2004JB003591, 2005.

Ide, S. and G. C. Beroza, Does apparent stress vary with earthquake size? Geophys. Res. Lett., 28, 3349-3352, 2001.

Kanamori, H. and D. L. Anderson, Theoretical basis of some empirical relations in seismology, Bull. Seismol. Soc. Am., 65, 1073-1095, 1975.

Kostrov, B. V., Seismic moment and energy of earthquakes and seismic flow of rock, Izv. Earth Phys., 1, 23-40, 1974.

Matsu'ura, M., H. Kataoka, and B. Shibazaki, Slip-dependent friction law and nucleation processes in earthquake rupture, Tectonophysics, 211, 135-148, 1992.

Ohnaka, M., A constitutive scaling law and a unified comprehension for frictional slip failure, shear fracture of intact rock, and earthquake rupture, J. Geophys. Res., 108, doi:10.1029/2000JB000123, 2003.

Okubo, P. G. and K. Aki, Fractal geometry in the San Andreas fault system, J. Geophys. Res., 92, 345-355, 1987.

Olsen, K. B., R. Madariaga, and R. J. Archuleta, Three-dimensional dynamic simulation of the 1992 Landers earthquake, Science, 278, 834 837, 1997.

Power, W. L., T. E. Tullis, S. R. Brown, G. N. Boitnott, and C. H. Scholz, Roughness of natural fault surfaces, Geophys. Res. Lett., 14, 29-32, 1987.

Renard, F., C. Voisin, D. Marsan, and J. Schmittbuhl, High resolution 3D laser scanner measurements of a strike-slip fault quantify its morphological anisotropy at all scales, Geophys. Res. Lett., 33, doi:10.1029/2005GL025038, 2006.

Ripperger, J., P. M. Mai, and J. P. Ampuero, Earthquake source characteristics from dynamic rupture with constrained stochastic fault stress, $J$. Geophys. Res., 112, doi:10.1029/2006JB004515, 2007. 
Sagy, A., E. E. Brodsky, and G. J. Axen, Evolution of fault-surface roughness with slip, Geology, 35, 283-286, 2007.

Stirling, M. W., S. G. Wesnousky, and K. Shimazaki, Fault trace complexity, cumulative slip, and the shape of the magnitude-frequency distribution for strike-slip faults: a global study, Geophys. J. Int., 124, 833-868, 1996.

Tada, T., Boundary integral equations for the time-domain and timeindependent analyses of 2D non-planar cracks, $\mathrm{PhD}$ Thesis, University of Tokyo, 1996.

Uenishi, K. and J. R. Rice, Universal nucleation length for slip-weakening rupture instability under nonuniform fault loading, J. Geophys. Res., 108, doi:10.1029/2001JB001681, 2003.
Walter, W. R., K. Mayeda, R. Gok, and A. Hofstetter, The scaling of seismic energy with moment; Simple models compared with observations, in Earthquakes: Radiated energy and the physics of faulting, AGU geophysical monograph 170, edited by R. Abercrombie, A. McGarr, H. Kanamori, G. DiToro, 25-41, 2006.

Yamada, T., J. J. Mori, S. Ide, R. E. Abercrombie, H. Kawakata, M. Nakatani, Y. Iio, and H. Ogasawara, Stress drops and radiated seismic energies of microearthquakes in a South African gold mine, J. Geophys. Res., 112, doi:10.1029/2006JB004553, 2007.

S. Ide (e-mail: ide@eps.s.u-tokyo.ac.jp) 